\title{
Energy dissipation by internal stresses in a free-rotating symmetric ellipsoid: Application to Comet P/Halley
}

\author{
A. Molina ${ }^{1,2}$, F. Moreno ${ }^{2}$, and F. Martínez-López ${ }^{1}$ \\ ${ }^{1}$ Departamento de Física Aplicada, University of Granada, C/ Fuentenueva s/n 18071 Granada, Spain \\ e-mail: fmartine@ugr.es \\ 2 Instituto de Astrofísica de Andalucía, CSIC, PO Box 3004, 18080 Granada, Spain \\ e-mail: fernando@iaa.es
}

Received 17 July 2002 / Accepted 5 November 2002

\begin{abstract}
Internal stresses dissipate energy in a rotating body, unless it spins about one of its principal axes. If the body is rotating around a non-principal axis, its major-inertia axis and its angular velocity will be nutating about the constant vector of angular momentum. In the course of time, the half-angles of the appropriate precession cones will be slowly shrinking due to dissipative effects. In this way, the body will be approaching the state of minimal kinetic energy. We establish here expressions for the components of the stress tensor in the case of a symmetric ellipsoid continuum body, which is a more suitable shape than the rectangular prism studied in earlier works. Using classical expressions of linear elasticity, we obtain the strain energy per unit volume and the temporal average of the total strain energy. In our model, the relative contribution from the doublefrequency mode to the inelastic-dissipation effect is larger than the contribution established by Lazarian \& Efroimsky (1999), Efroimsky \& Lazarian (2000), and Efroimsky (2001) for a prism. We come up with an expression for the relaxation time and compare it with similar expressions previously derived in the literature. In terms of the overall intensity of the effect, our result is in between the result derived in Lazarian \& Efroimsky (1999), Efroimsky \& Lazarian (2000), and Efroimsky (2002) and that in Burns \& Safronov (1973). We apply our model to the comet P/Halley. Following the model proposed by Belton et al. (1991) for the rotation of Halley's comet, we estimate a relaxation time of $10^{2}$ to $10^{4}$ million years depending on the quality factor assumed.
\end{abstract}

Key words. comets: general - comets: individual: P/Halley

\section{Introduction}

Rotation is a common feature of unsupported objects. In particular, comets exhibit rotation which, in many cases, is complex (i.e. about axes different from principal). Observation and theoretical analysis of cometary rotation is an important area of astronomical research, because it enables us to establish a connection between the dynamical parameters of the comets with their physical properties. In order to explain the observational fact that asteroids have singly-periodic light curves, Prendergast (1958) presented a theory whereby the internal friction dissipates kinetic energy, thus the asteroids, maintaining constant the angular momentum, will ultimately reach the minimum energy state, i.e. when the axis of rotation has the same direction as the principal axis of inertia. In fact, the theory of Prendergast is based in the classical theory of linear elasticity and its main conclusion is that the asteroids with single period light curves have not suffered important collisions during the time of the alignment of the mentioned above axis, which was calculated to be less than a few million years.

Send offprint requests to: A. Molina,

e-mail: molina@iaa.es, amolina@ugr.es
Burns \& Safronov (1973) also derived approximate expressions for the alignment time and their results showed that the internal energy dissipation is a relatively rapid mechanism. Independent of those early attempts, Purcell (1979) addressed the problem of nutation dissipation in the context of interstellar-dust research. He studied suprathermal (with energies exceeding $\left.(3 / 2) k T_{\text {gas }}\right)$ complex rotation of the granules, and came to the conclusion that their relaxation rate is an important parameter regulating the process of dust alignment relative to the interstellar magnetic field. This resulted in the necessity of estimating the relaxation rate. Purcell considered two mechanisms of kinetic-energy dissipation. One was the so-called Barnett dissipation (dissipation resulting from nutation-caused periodic remagnetisation of a paramagnetic granule). Another was inelastic dissipation, i.e., inner friction caused by nutation-caused alternating stresses and strains. Similarly to Burns \& Safronov (1973), Purcell (1979) missed the generation of harmonics. He also miscalculated the distribution of stresses. As a result, he badly underestimated the relevance of inelastic-dissipation effects. A more accurate theory for prism-shaped oblate granules was developed by Lazarian \& Efroimsky (1999), hereafter L\&E. Later, those authors extended their approach to wobbling 
asteroids and comets (Efroimsky \& Lazarian 2000; Efroimsky 2000, 2001, 2002). On the one hand, the theory of Lazarian \& Efroimsky was developed for prism-shaped bodies solely. On the other hand, even for that shape, they provided only approximate expressions for the stress and strain within the body. Our intention is to consider a more realistic shape, that of an oblate symmetric ellipsoid and to offer an improved solution for the stress in this model.

Although many studies on various precessing bodies have been made, only a few small bodies show a complex rotational state, and undoubtedly, concerning comets, Comet Halley, which is a well studied comet, is clearest example of a comet showing precession in its rotation.

In Sect. 2 we establish the basis for a dynamical study of a rotating body assuming a non-rigid behavior. Once having obtained new expressions for the components of the stress tensor of a symmetric ellipsoid with the appropriate boundary conditions, we use linear elasticity theory to get the equation for the average time value of the elastic potential in Sect. 3, as well as the rate of alignment, Sect. 4. In Sect. 5, we present the discussion of our results and a comparison with those reported by other authors. The conclusions appear in Sect. 6. The details of the determination of the pertinent equations are given in the Appendix.

\section{Basic ideas and notation}

Rotation of a rigid body can be studied by the Euler equations (see any Classical Mechanics book: for instance, Marion 1970), and if the body is assumed to be an isolated system, the angular momentum, $\boldsymbol{L}$, is constant.

Briefly, two reference systems are considered: an inertial frame ( $X, Y, Z$ rectangular coordinates) that has the $Z$ axis in the $\boldsymbol{L}$ (constant) direction, and a body system $(x, y, z$ rectangular coordinates). The body is assumed to be a symmetric ellipsoid (semi-axes $a=b \neq c$ ) whose principal moments of inertia are denoted by $I$ (that refer to semi-axes $a$ and $b$ ) and by $I_{3}$ (that refer to semi-axis $c$ ), and it is rotating with an angular velocity $\mathbf{\Omega}$. The angle between this angular velocity vector and the $z$-axis (of the body system) is denoted by $\alpha$, and $\theta$ denotes the angle between the $z$-axis and $\boldsymbol{L}$. The body will be precessing (around the body symmetry axis for an observer in the body system and around the $Z$-axis, or $\boldsymbol{L}$, for an observer in the inertial system) with a constant angular velocity given by $\omega=(h-1) \Omega_{3}$, where $h=\frac{I_{3}}{I}$. Thus, the projection of $\boldsymbol{\Omega}$ over the $x-y$ plane, $\Omega_{\perp}$, describes a circumference over that plane. As the body is torque- free, the kinetic rotational energy is constant unless a dissipative process is present. We assume that small deformations occur in the interior of the body, the dissipative mechanism being what transforms part of the kinetic energy of rotation in to elastic energy potential. Then, $\boldsymbol{\Omega}$ tends to have the same direction as the symmetry axis of the maximum moment of inertia, and when this occurs the system has reached its state of minimum energy (the most stable state). What is the cause of those small strains? As is known, strains are a consequence of stresses and these appear because particles of the body suffer an acceleration in the inertial frame, consisting of two terms: that known as centrifugal force and that due to the time variation of $\boldsymbol{\Omega}$ (see appendix).

Our treatment of the problem will be based on the adiabatic approximation. This approach, used previously by Efroimsky \& Lazarian (2000) and Efroimsky (2001, 2002), rests on the presence of two separate time scales in this problem: typically (though not always), the rotation and nutation are much faster processes than nutation relaxation. We shall dwell on this in the end of Sect. 4. Beside adiabaticity, we shall introduce one more physically reasonable assumption (which, too, was tacitly used in the aforementioned papers). On the one hand, when considering the interior stresses and strains, we shall assume that the body is deformable. On the other hand, when imposing boundary conditions on the stresses, we shall assume that the boundaries' shape are stiff, as if the body were absolutely rigid. This treatment seems acceptable for two reasons. First, the wobble is extremely slow, and the frequencies involved are much lower than the acoustic eigenfrequencies of the body. Second, modelling a comet or asteroid by a homogeneous ellipsoid is by itself quite a rough approximation, and the deviation of realistic shapes from an ideal ellipsoid will much exceed the small variations of the free boundary.

\section{Elastic potential}

Although there are excellent books on continuum mechanics (for example, Sedov 1971), we think it opportune to provide here a very brief introduction to the strain theory before considering the elastic potential. Two tensors play a fundamental role: the strain tensor, $\epsilon$, and the stress tensor, $\tau$. The former gives information on the deformation produced in a non-rigid body (the effect) when a stress is applied to it, which is expressed by the stress tensor (the cause). Because tensors are abstract mathematical entities, we are going to apply them to the case in which they can be expressed by simple scalar concepts. Let $\mathrm{d} s$ be the modulus of the $\mathrm{d} \boldsymbol{r}(\boldsymbol{r}$ is the radius vector at time $t)$, and ds' the same at time $t^{\prime}$, we define the coefficient of relative elongation, denoted by $l$, as $l=\frac{\mathrm{d} s-\mathrm{d} s^{\prime}}{\mathrm{d} s}$. It depends on the point of the body and of the considered direction. When the considered directions are those of the principal components of the $\epsilon_{i j}$, then $l_{i}=\left(1+\frac{2 \epsilon_{i i}}{g_{i i}^{0}}\right)^{\frac{1}{2}}-1\left(g_{i j}^{0}\right.$ are the components of the metric tensor an instant 0 ). If the deformations are small, then the $\epsilon_{i i}$ are small, and expanding the previous expression in a power series, one finds $l_{i} \approx \frac{\epsilon_{i i}}{g_{i i}^{0}}$. Besides, if one selects a Cartesian coordinate system, $g_{i i}^{0}=0$ and, consequently, $l_{i}=\epsilon_{i i}$. Thus, a very simple example is a rod subjected to a tensile force (in the rod direction), the strain is the change in length over the original length (assuming the increase in length is small) which, evidently, is dimensionless. On the other hand, the stress tensor is here the applied force per unit cross-sectional area of the rod, with the dimension of pressure. Evidently, both quantities are connected by physical parameters which depend on the material considered. In the simple case shown above, we can apply the wellknown Hooke's law: "ut tensio sic vis", which can be translated freely as "the extension is proportional to the force". In the example shown, the strain and the stress are related by the known Young modulus. Lastly in this introduction, we show the 
concept of the displacement vector, $\boldsymbol{u}_{i}$, as $\boldsymbol{u}_{i}=\boldsymbol{r}_{i}-\boldsymbol{r}_{i}^{0}, \boldsymbol{r}_{i}$ and $\boldsymbol{r}_{\boldsymbol{i}}^{0}$ being the radius vector of the $i$ particle in the instant $t$ and $t^{0}$, respectively. When a Euclidean space is used (which occurs in the practical cases) this displacement function is useful because its spatial derivative plays the role of the strain.

The elastic potential, also called volume density of strain energy, $\ni$, plays an important role in our study. (From the point of view of thermodynamics it really exists only if the strain process occurs very fast or very slowly.) As $\ni=\frac{1}{2} \varepsilon_{i j} \tau^{i j}$, being $\varepsilon_{i j}$ the components of the strain tensor and $\tau^{i j}$ the components of the stress tensor (see e.g., Sedov 1971; Sokolnikoff 1956) and considering the relation between both components ${ }^{1}$ $\tau_{i j}=\lambda \varepsilon_{m m} \delta_{i j}+2 \mu \varepsilon_{i j}$, which assumes an isotropic linear elastic behavior, $\ni$ can be expressed in terms of $\varepsilon_{i j}$ ( $\lambda$ and $\mu$ are the Lamé constants, and they are related to the known Young's modulus and Poisson ratio). As $\varepsilon_{i j}=\frac{1}{2}\left(\frac{\partial u_{i}}{\partial x_{j}}+\frac{\partial u_{j}}{\partial x_{i}}\right)$, $\ni$ can be considered as a function of the components $u_{i}$ of the displacement vector. The Navier equation of the Linear Elasticity (in the equilibrium) is:

$(\lambda+\mu) \operatorname{grad} \operatorname{div} \boldsymbol{u}+\mu \Delta \boldsymbol{u}+\rho \boldsymbol{F}=0$,

which is written in Cartesian coordinates as:

$(\lambda+\mu) \frac{\partial}{\partial x_{i}}\left(\frac{\partial u_{k}}{\partial x_{k}}\right)+\mu \frac{\partial^{2} u_{k}}{\partial x_{k}^{2}}+\rho F_{i}=0$,

$F_{i}$ being the component $i$ of the body forces per unit mass. If only centrifugal forces are present, $\rho F_{i}=\rho\left(\Omega^{2} x_{i}-\Omega_{i} \Omega_{k}\right)$. Thus, solving the Navier equation and taking into account the boundary conditions, the components $u_{i}$ are obtained. Knowing $u_{i}$, $\varepsilon_{i j}$ is immediately derived, and then, $\ni$ is finally obtained. This way to get the strain energy per unit volume is elegant but not easy. Prendergast (1958) used a similar way to obtain $\ni$ and the volume integral of the time dependent part of the elastic potential, which he called the energy of vibration, but in what concerns his statement "the solution of this equation and the computation of the strain energy function will be given elsewhere", as he wrote, it is unfortunately unknown to us where this information was published.

Here we have preferred to obtain $\ni$ in the way employed by L\&E. Thus, our purpose is to get the stress tensor components.

The equilibrium equation (from the continuum equation of motion) is $\rho F_{i}=-\frac{\partial \tau_{i j}}{\partial x_{j}}$. Then, we can write, in matrix form

$$
\left(\begin{array}{lll}
\frac{\partial}{\partial x} & \frac{\partial}{\partial y} & \frac{\partial}{\partial z}
\end{array}\right)\left(\begin{array}{lll}
\tau_{x x} & \tau_{x y} & \tau_{x z} \\
\tau_{y x} & \tau_{y y} & \tau_{y z} \\
\tau_{z x} & \tau_{z y} & \tau_{z z}
\end{array}\right)=\rho\left(\begin{array}{lll}
a_{x} & a_{y} & a_{z}
\end{array}\right)
$$

$a_{i}$ being the components of the acceleration due to the centrifugal force in the rotation. We need only six components as the stress tensor is a symmetric tensor, and all of them must vanish on the boundaries of the symmetric paraboloid, $\frac{x^{2}}{a^{2}}+\frac{y^{2}}{a^{2}}+\frac{z^{2}}{c^{2}}=1$. Thus, we establish the following expressions:

$\tau_{x x}=\frac{\rho}{4} \Omega_{\perp}^{2}\left[x^{2}-\left(a^{2}-\frac{a^{2}}{c^{2}} z^{2}-y^{2}\right)\right] \cos 2 \omega t$

\footnotetext{
${ }^{1}$ Because Cartesian coordinates are used, distinction between contravariant and covariant components will not be made.
}

$$
\begin{aligned}
\tau_{x y}= & \frac{\rho}{4} \Omega_{\perp}^{2}\left[y^{2}-\left(a^{2}-\frac{a^{2}}{c^{2}} z^{2}-x^{2}\right)\right] \sin 2 \omega t \\
\tau_{x z}= & \frac{\rho}{2} \Omega_{\perp} \Omega_{3} h\left[z^{2}-\left(a^{2}-x^{2}-y^{2}\right) \frac{c^{2}}{a^{2}}\right] \cos \omega t \\
\tau_{y y}= & -\frac{\rho}{4} \Omega_{\perp}^{2}\left[y^{2}-\left(a^{2}-\frac{a^{2}}{c^{2}} z^{2}-x^{2}\right)\right] \cos 2 \omega t \\
\tau_{y z}= & \frac{\rho}{2} \Omega_{\perp} \Omega_{3} h\left[z^{2}-\left(a^{2}-x^{2}-y^{2}\right) \frac{c^{2}}{a^{2}}\right] \sin \omega t \\
\tau_{z z}= & \rho \Omega_{\perp} \Omega_{3}\left(2-h \frac{c^{2}}{a^{2}}-h\right)(x \cos \omega t+y \sin \omega t) \\
& \times\left\{z-\left[\left(a^{2}-x^{2}-y^{2}\right) \frac{c^{2}}{a^{2}}\right]^{\frac{1}{2}}\right\}
\end{aligned}
$$

It is easy to show that all of them vanish on the ellipsoid boundary, as well as getting the components of the acceleration by simple derivation (see appendix):

$$
\begin{aligned}
& a_{x}=\frac{1}{2} \Omega_{\perp}^{2} x \cos \omega t+\frac{1}{2} \Omega_{\perp}^{2} y \sin \omega t+z \Omega_{\perp} \Omega_{3} h \cos \omega t \\
& a_{y}=\frac{1}{2} \Omega_{\perp}^{2} x \sin 2 \omega t-\frac{1}{2} \Omega_{\perp}^{2} y \cos \omega t+z \Omega_{\perp} \Omega_{3} h \sin \omega t \\
& a_{z}=\Omega_{\perp} \Omega_{3}(2-h)(x \cos \omega t+y \sin \omega t) .
\end{aligned}
$$

In fact, we only consider the temporal part of the acceleration and of the stress tensor, because only those terms influence the rate of energy dissipation.

As is well known from the theory of elasticity, Eq. (3) is a necessary condition for determining the picture of stresses. Another condition which the stress tensor must obey is that of symmetry: $\tau_{i j}=\tau_{j i}$. Finally, the tensor should satisfy the standard boundary conditions:

$\tau_{i j} n_{j}=0$,

$\boldsymbol{n}$ being the unit vector normal to the surface. All these necessary conditions, however, are not yet sufficient for determining the stress distribution over the volume. The remaining freedom is removed by the so-called supplimentary conditions imposed on $\tau_{i j}$. These conditions (explained, for example, in Durelli et al. 1958) are used for elastic media. They makes our problem very mathematically involved. To avoid excessive complications, we substitute these supplimentary conditions by another one: we assume that the tangential components of the stress vanish over the boundary. (Along with the standard boundary condition (13), this supplimentary condition leads to the assumption that the stresses completely vanish on the boundary.) Since our supplimentary condition is different from the right one, our expressions (4)-(9) furnish not a rigorous solution but another approximation thereto. We believe that this approximation is better than that used by Lazarian \& Efroimsky (1999) and (Efroimsky \& Lazarian 2000), because our solution obeys (13) exactly, while the solution offered in the said papers obeyed (13) only approximately.

The presence of only two frequencies in the components of stress tensor (and hence in the elastic potential) results from the dynamical oblate symmetry of the body: the two largest semiaxes of the ellipsoid coincide, wherefrom the moments of inertia obey relation $I_{3}>I_{2}=I_{1}$. Though this treatment cannot be 
extended to the case of a dynamically prolate symmetrical rotator, we intend to apply our results to an elongated body, comet $\mathrm{P} /$ Halley, with the assumption that these results may serve at least as an estimate. We shall say more on this topic in Sect. 5.

Once the stress tensor has been established, we can obtain the strain energy per unit volume. The components of strain tensor can be expressed in terms of the components of the stress tensor by the known relation

$\varepsilon_{i j}=-\frac{\lambda}{2 \mu(3 \lambda+2 \mu)} \delta_{i j} I_{1}(\tau)+\frac{1}{2 \mu} \tau_{i j}$,

where $I_{1}(\tau)$ is the first invariant of the stress tensor. Then, after substituting in $\ni=\frac{1}{2} \varepsilon_{i j} \tau_{i j}$ we have

$\ni=-\frac{\lambda}{4 \mu(3 \lambda+2 \mu)} \delta_{i j} I_{1}(\tau) \tau_{i j}+\frac{1}{4 \mu} \tau_{i j} \tau_{i j}$

As $I_{1}(\tau)=\sum_{i} \tau_{i i}=\tau_{x x}+\tau_{y y}+\tau_{z z}$, $\ni$ becomes

$$
\begin{aligned}
\ni= & \frac{1}{4 \mu}\left[-\frac{\lambda}{(3 \lambda+2 \mu)} \delta_{i j} \tau_{k k} \tau_{i j}+\tau_{i j} \tau_{i j}\right] \\
= & \frac{1}{4 \mu}\left[-\frac{\lambda}{(3 \lambda+2 \mu)}\left(\tau_{x x}+\tau_{y y}+\tau_{z z}\right)^{2}+\tau_{x x}^{2}+\tau_{y y}^{2}\right. \\
& \left.+\tau_{z z}^{2}+2 \tau_{x y}^{2}+2 \tau_{x z}^{2}+2 \tau_{y z}^{2}\right] .
\end{aligned}
$$

Finally, considering the relation between the Poisson ratio, $\sigma$, and the Lamé parameters, $\sigma=\frac{\lambda}{2(\lambda+\mu)}$, we establish that

$$
\begin{aligned}
\ni= & \frac{1}{4 \mu}\left[-\frac{\sigma}{1+\sigma}\left(\tau_{x x}+\tau_{y y}+\tau_{z z}\right)^{2}+\tau_{x x}^{2}+\tau_{y y}^{2}\right. \\
& \left.+\tau_{z z}^{2}+2 \tau_{x y}^{2}+2 \tau_{x z}^{2}+2 \tau_{y z}^{2}\right] .
\end{aligned}
$$

The next step is the calculation of the temporal average of the total strain energy, $\langle W\rangle$. The time average of the elastic potential is given by:

$$
\langle W\rangle=\frac{1}{T} \int_{0}^{T} \mathrm{~d} t \int_{V} \ni \mathrm{d} v,
$$

$T$ being the precession period and $V$ all the volume of the body. After substituting $\ni$ by the above expression, we have

$$
\begin{aligned}
\langle W\rangle= & \frac{\omega}{2 \pi} \frac{1}{4 \pi} \int_{0}^{\frac{\omega}{2 \pi}}\left\{\int _ { V } \frac { 1 } { 4 \mu } \left[-\frac{\sigma}{1+\sigma}\left(\tau_{x x}+\tau_{y y}+\tau_{z z}\right)^{2}+\tau_{x x}^{2}\right.\right. \\
& \left.\left.+\tau_{y y}^{2}+\tau_{z z}^{2}+2 \tau_{x y}^{2}+2 \tau_{x z}^{2}+2 \tau_{y z}^{2}\right] \mathrm{~d} v\right\} \mathrm{d} t
\end{aligned}
$$

Now, calling $s_{i j}$ the time-independent part (spatial part) of $\tau_{i j}$ and taking into account the following results:

$$
\begin{aligned}
& \int_{0}^{\frac{\omega}{2 \pi}} \sin ^{3} \omega t \mathrm{~d} t=\int_{0}^{\frac{\omega}{2 \pi}} \cos ^{3} \omega t \mathrm{~d} t=\int_{0}^{\frac{\omega}{2 \pi}} \cos \omega t \sin \omega t \mathrm{~d} t=0 \\
& \int_{0}^{\frac{\omega}{2 \pi}} \cos ^{2} \omega t \sin \omega t \mathrm{~d} t=\int_{0}^{\frac{\omega}{2 \pi}} \sin ^{2} \omega t \cos \omega t \mathrm{~d} t \\
& \quad=\int_{0}^{\frac{\omega}{2 \pi}} \cos 2 \omega t \sin \omega t \mathrm{~d} t=0
\end{aligned}
$$

$$
\begin{aligned}
& \int_{0}^{\frac{\omega}{2 \pi}} \cos 2 \omega t \cos \omega t \mathrm{~d} t=\int_{0}^{\frac{\omega}{2 \pi}} \cos 2 \omega t \sin 2 \omega t \mathrm{~d} t \\
& =\int_{0}^{\frac{\omega}{2 \pi}} \cos 2 \omega t \sin \omega t \cos \omega t \mathrm{~d} t=0 \\
& \int_{0}^{\frac{\omega}{2 \pi}} \sin ^{2} \omega t \mathrm{~d} t=\int_{0}^{\frac{\omega}{2 \pi}} \cos ^{2} \omega t \mathrm{~d} t=\frac{\pi}{\omega} \\
& \int_{0}^{\frac{\omega}{2 \pi}} \cos 2 \omega t \cos ^{2} \omega t \mathrm{~d} t=\frac{3}{2} \frac{\pi}{\omega} \\
& \int_{0}^{\frac{\omega}{2 \pi}} \cos 2 \omega t \sin ^{2} \omega t \mathrm{~d} t=-\frac{3}{2} \frac{\pi}{\omega}
\end{aligned}
$$

we can write

$$
\begin{aligned}
\langle W\rangle= & \frac{\omega}{2 \pi} \frac{1}{4 \pi} \int_{V}\left[-\frac{\sigma}{1+\sigma}\left\{s_{x x}^{2} \frac{\pi}{\omega}+s_{y y}^{2} \frac{\pi}{\omega}\right.\right. \\
& \left.+s_{z z}^{2}\left(x^{2} \frac{\pi}{\omega}+y^{2} \frac{\pi}{\omega}\right)+2 s_{x x} s_{y y} \frac{\pi}{\omega}\right\}+s_{x x}^{2} \frac{\pi}{\omega}+s_{y y}^{2} \frac{\pi}{\omega} \\
& \left.+s_{z z}^{2}\left(x^{2} \frac{\pi}{\omega}+y^{2} \frac{\pi}{\omega}\right)+2 s_{x y}^{2} \frac{\pi}{\omega}+2 s_{x z}^{2} \frac{\pi}{\omega}+2 s_{y z}^{2} \frac{\pi}{\omega}\right] \mathrm{d} v .
\end{aligned}
$$

The last expression can be rewritten as

$$
\begin{aligned}
\langle W\rangle= & \left\langle W^{(\omega)}\right\rangle+\left\langle W^{(2 \omega)}\right\rangle \\
= & \frac{1}{8 \mu} \int_{V}\left(\frac{s_{z z}^{2}}{1+\sigma}\left(x^{2}+y^{2}\right)+2 s_{x z}^{2}+2 s_{y z}^{2}\right) \mathrm{d} v \\
& +\frac{1}{8 \mu} \int_{V}\left(\frac{s_{x x}^{2}+s_{y y}^{2}}{1+\sigma}+\frac{-\sigma}{1+\sigma} 2 s_{x x} s_{y y}+2 s_{x y}^{2}\right) \mathrm{d} v,
\end{aligned}
$$

where we have two separate terms, one, $\left\langle W^{(\omega)}\right\rangle$, indicating the part due to the simple frequency and the other, $\left\langle W^{(2 \omega)}\right\rangle$, produced by double frequency. Next, we calculate both terms.

As the body is an ellipsoid, $2-h \frac{c^{2}}{a^{2}}-h=0$, and then $\tau_{z z}=$ $s_{z z}=0$. Therefore,

$$
\begin{aligned}
\left\langle W^{(\omega)}\right\rangle & =\frac{1}{8 \mu} \int_{V}\left(2 s_{x z}^{2}+2 s_{y z}^{2}\right) \mathrm{d} v \\
& =\frac{h^{2} \rho^{2} \Omega_{\perp}^{2} \Omega_{3}^{2}}{16 \mu} \int_{V}\left[z^{2}-\left(a^{2}-x^{2}-y^{2}\right) \frac{c^{2}}{a^{2}}\right]^{2} \mathrm{~d} v .
\end{aligned}
$$

To resolve the integration we make a change of variables: $x^{\prime}=x / a ; y^{\prime}=y / a ; z^{\prime}=z / c$. Then,

$$
\left\langle W^{(\omega)}\right\rangle=\frac{h^{2} \rho^{2} \Omega_{\perp}^{2} \Omega_{3}^{2} a^{2} c^{5}}{16 \mu} \int_{V^{\prime}}\left(x^{\prime 2}+y^{\prime 2}+z^{\prime 2}-1\right)^{2} \mathrm{~d} v,
$$

and choosing spherical coordinates:

$$
\left\langle W^{(\omega)}\right\rangle=\frac{h^{2} \rho^{2} \Omega_{\perp}^{2} \Omega_{3}^{2} a^{2} c^{5}}{16 \mu} \int_{0}^{2 \pi} d \varphi \int_{0}^{\pi} \sin \theta d \theta \int_{0}^{1}\left(r^{2}-1\right)^{2} r^{2} \mathrm{~d} r
$$

which gives

$\left\langle W^{(\omega)}\right\rangle=\frac{2 \pi h^{2} \rho^{2} \Omega_{\perp}^{2} \Omega_{3}^{2} a^{2} c^{5}}{105 \mu}$,

Finally, as $\Omega_{3}=\Omega_{0} \cos \theta, \Omega_{\perp}=\Omega_{0} h \sin \theta$, and $h=\frac{2}{1+\frac{c^{2}}{a^{2}}}$, we establish that

$\left\langle W^{(\omega)}\right\rangle=\frac{32 \pi \rho^{2} \Omega_{0}^{4} \sin ^{2} \theta \cos ^{2} \theta}{105 \mu} \frac{a^{10} c^{5}}{\left(a^{2}+c^{2}\right)^{4}}$. 
The other contribution to the strain energy, which is due to the double frequency $2 \omega$, is

$$
\left\langle W^{(2 \omega)}\right\rangle=\frac{1}{8 \mu} \int_{V} \mathrm{~d} V\left(\frac{s_{x x}^{2}+s_{y y}^{2}}{1+\sigma}+\frac{-\sigma}{1+\sigma} 2 s_{x x} s_{y y}+2 s_{x y}^{2}\right) \text {. }
$$

After substituting $s_{i j}$ expressions, we have

$$
\begin{aligned}
& \left\langle W^{(2 \omega)}\right\rangle=\frac{\Omega_{\perp}^{4}}{64 \mu} \int_{V} \mathrm{~d} V\left(\frac { 1 } { 2 ( 1 + \sigma ) } \left\{\left[x^{2}-\left(a^{2}-\frac{a^{2}}{c^{2}} z^{2}-y^{2}\right)\right]^{2}\right.\right. \\
& \left.+\left[y^{2}-\left(a^{2}-\frac{a^{2}}{c^{2}} z^{2}-x^{2}\right)\right]^{2}\right\}+\frac{-\sigma}{1+\sigma}\left[x^{2}-\left(a^{2}-\frac{a^{2}}{c^{2}} z^{2}-y^{2}\right)\right] \\
& \left.\quad \times\left[y^{2}-\left(a^{2}-\frac{a^{2}}{c^{2}} z^{2}-x^{2}\right)\right]+\left[y^{2}-\left(a^{2}-\frac{a^{2}}{c^{2}} z^{2}-x^{2}\right)\right]^{2}\right] \cdot
\end{aligned}
$$

Following the same method as for the previous part $\left\langle W^{(\omega)}\right\rangle$, after a variable change is made and using spherical coordinates, we finally obtain

$$
\left\langle W^{(2 \omega)}\right\rangle=\frac{16 \pi \rho^{2} \Omega_{0}^{4} \sin ^{4} \theta}{105 \mu(1+\sigma)} \frac{a^{14} c}{\left(a^{2}+c^{2}\right)^{4}} .
$$

\section{Alignment rate}

As said before, once the average total strain energy is known, the next step is to calculate how fast that elastic potential energy is dissipated. If we denote the rotation energy by $E$, its time derivative will be $\frac{\mathrm{d} E}{\mathrm{~d} t}=\frac{\mathrm{d} E}{\mathrm{~d} \theta} \frac{\mathrm{d} \theta}{\mathrm{d} t}$, and therefore we have $\frac{\mathrm{d} \theta}{\mathrm{d} t}=\frac{\frac{\mathrm{d} E}{\mathrm{~d} t}}{\frac{\mathrm{d} \theta}{\mathrm{d} \theta}}$. As the time variation of the rotation energy is assumed to be equal to the time variation of the strain energy, and $\frac{\mathrm{d} E}{\mathrm{~d} \theta}=\omega L \sin \theta$, we can establish that $\frac{\mathrm{d} \theta}{\mathrm{d} t}=\frac{\frac{\mathrm{d} W}{\mathrm{~d} t}}{\omega L \sin \theta}$. As $\frac{\mathrm{d}\langle W\rangle}{\mathrm{d} t}=\frac{\mathrm{d}\left\langle W^{(\omega)}\right\rangle}{\mathrm{d} t}+\frac{\mathrm{d}\left\langle W^{(2 \omega)}\right\rangle}{\mathrm{d} t}$ and the energy rate is related with the quality factor, $Q$, by means of the equation $\frac{\mathrm{d} W}{\mathrm{~d} t}=-\omega \frac{W}{Q}$, we can obtain $\frac{\mathrm{d} \theta}{\mathrm{d} t}$ as a function of $\omega, Q$ and $W$. Efroimsky \& Lazarian (2000) modified in a factor of 2 the appropriate expression of L\&E and obtained the expression

$\frac{\mathrm{d} \theta}{\mathrm{d} t}=-\frac{3}{16} \frac{a^{2} \Omega_{0}^{3} \rho}{\mu Q} \frac{63\left(\frac{c}{a}\right)^{4} \operatorname{ctg}^{2} \theta+20}{\left(1+\frac{c^{2}}{a^{2}}\right)^{4}} \sin ^{3} \theta$

after assuming a Poisson rate of 0.25 . The term containing $63\left(\frac{c}{a}\right)^{4} \operatorname{ctg}^{2} \theta$ is related to the simple frequency mode and the term containing the factor of 20 is related to the double frequency mode.

As a comparison with the results reported by Efroimsky \& Lazarian (2000) will be made in the next section, we will use here the same expression as they employed, i.e. $\frac{\mathrm{d} W}{\mathrm{~d} t}=$ $-2 \frac{\omega}{Q}\left[W^{(\omega)}+2 W^{(2 \omega)}\right]$, as $W^{(\omega)}$ and $2 W^{(2 \omega)}$ are considered average values. Then, and considering our expressions for $\left\langle W^{(\omega)}\right\rangle$ and $\left\langle W^{(2 \omega)}\right\rangle$, we write

$$
\begin{aligned}
\frac{\mathrm{d} \theta}{\mathrm{d} t}= & -\frac{2}{Q L \sin \theta}\left[\frac{32 \pi \rho^{2} \Omega_{0}^{4} \sin ^{2} \theta \cos ^{2} \theta}{105 \mu} \frac{a^{10} c^{5}}{\left(a^{2}+c^{2}\right)^{4}}\right. \\
& \left.+2 \frac{16 \pi \rho^{2} \Omega_{0}^{4} \sin ^{4} \theta}{105 \mu(1+\sigma)} \frac{a^{14} c}{\left(a^{2}+c^{2}\right)^{4}}\right] \\
= & -\frac{64 \pi a^{10} c \rho^{2} \Omega_{0}^{3}}{105 \mu\left(a^{2}+c^{2}\right)^{4} I_{3} Q}\left(c^{4} \sin \theta \cos ^{2} \theta+\frac{a^{4}}{1+\sigma} \sin ^{3} \theta\right),
\end{aligned}
$$

and as

$$
I_{3}=\frac{1}{5} M\left(2 a^{2}\right)=\frac{8}{15} \pi \rho a^{4} c,
$$

the expression becomes

$$
\frac{\mathrm{d} \theta}{\mathrm{d} t}=-\frac{8 a^{6} \rho \Omega_{0}^{3}}{7 \mu\left(a^{2}+c^{2}\right)^{4} Q}\left(c^{4} \sin \theta \cos ^{2} \theta+\frac{a^{4}}{1+\sigma} \sin ^{3} \theta\right) .
$$

The first term is related to the principal frequency and the second one corresponds to the double frequency mode.

From the previous equation, is easy to see that the alignment rate does not vanish when $\theta$ is equal to $\frac{\pi}{2}$, and hence, the equation is valid only if $\theta$ is not in the vicinity of $\theta=\frac{\pi}{2}$, region where the adiabatic approximation is not satisfied. An excellent discussion about this point can be seen in Efroimsky (2001).

\section{Results and discussion}

Before discussing the important results on the alignment rate and comparing them with those reported by other authors, we analyze the importance of each mode (principal frequency $\omega$ and double frequency mode $2 \omega$ ) which are present in the strain energy. We make such an analysis because L\&E emphasized the presence of the $2 \omega$ mode. They asserted that deformations at double frequency were disregarded in earlier studies, referring to Purcell (1979) while later Efroimsky \& Lazarian (2000) mentioned Burns \& Safronov (1973) too. In fact, in the last mentioned paper, its authors made a simple approximation considering the body as a bending of a slender beam, and hence, they used the most simple expressions of the elementary elasticity theory. In what concerns the Purcell paper, its author gave a mistaken expression for the acceleration, as it can be proved from the expression relating the acceleration between an inertial system and a non-inertial system (see a Classical Mechanics book, for instance Marion 1970). Thus, in Eq. (28) of Purcell (1979) the terms: $-x \Omega_{3}^{2}+y \Omega_{\perp}^{2} \sin \omega t \cos \omega t$ in the first component of the acceleration vector, $-y \Omega_{3}^{2}+x \Omega_{\perp}^{2} \sin \omega t \cos \omega t$ in the second component, and $-z \Omega_{3}^{2}$ in the third component of the acceleration, are missing (see appendix). It should be noted that in the first and in the second component a time-dependent term appears, which is an input in the stress tensor, and hence in the strain density energy.

If we make the ratio between the contribution to the strain energy owing to the simple frequency mode and that from the double frequency using the L\&E expressions (Poisson ratio equal 0.25$)$, then

$$
\frac{W^{(\omega)}}{2 W^{(2 \omega)}}=3.15\left(\frac{c}{a}\right)^{4} \cot ^{2} \theta
$$

However, using the equations obtained by us, the ratio becomes

$$
\frac{W^{(\omega)}}{2 W^{(2 \omega)}}=1.25\left(\frac{c}{a}\right)^{4} \cot ^{2} \theta .
$$

Therefore, the relative importance of the double frequency mode is even greater in the case of the an ellipsoidal body (using our expressions) than that noted by L\&E by a factor of 2.5 .

Once the rate of alignment is obtained, a simple integration gives us the time required for the maximal inertia axis of the 


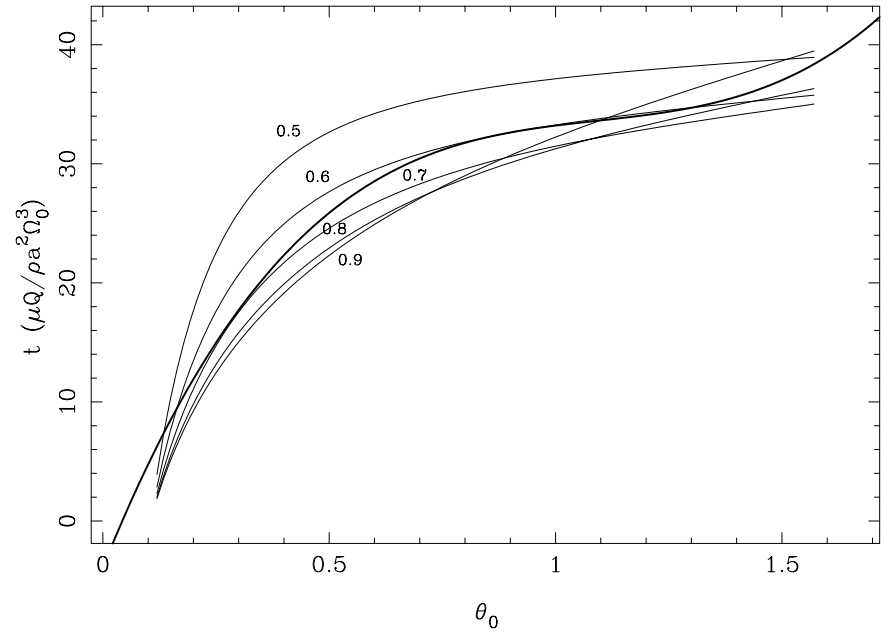

Fig. 1. Relaxation time for different $c / a$ ratio (thin lines) and fitted curve (thick line) versus initial angle $\theta_{0}$ (see text).

body to be shifted towards alignment with the angular moment. In order to avoid the divergence when the $\theta$ values are near zero, a non-null value of that angle for the upper limit in the integral has been chosen. L\&E chose a value of $6^{\circ}$ for such angle, and we will adopt the same value in order to compare results. The evaluation of this relaxation time is one of the purposes of this work, Therefore, before going into a discussion on the values of the constants $\rho, \mu$ and $Q$, it is appropriate to compare the results obtained by other authors with our result of relaxation time.

The time of alignment can be obtained by integration of the alignment rate. In Fig. 1 we show the relaxation time (scaled by a factor $\mu Q / \rho a^{2} \Omega_{0}^{3}$ ) as a function of $\theta_{0}$ (initial angle) for different values of the semi-axis ratio. Figure 1 also shows a thirdorder polynomial $t=25.25 \theta_{0}^{3}-82.79 \theta^{2}+94.66 \theta-3.9$ which is a fit to the curves shown (standard deviation 2.9). The first result of the comparison with the analogous curves by Efroimsky \& Lazarian (2000) is that the relaxation time is greater in our case than that reported by the aforequoted authors, by a factor close to 8 . Nevertheless, as a prism of halfsizes $a \times b \times c$, is approximately twice heavier than an ellipsoid of semi-axes $a \times b \times c$, the said factor will not be 8 , but between 4 and 5 . Now, if we compare this result with that given by Burns $\&$ Safronov (1973), $t=100\left(\mu Q / \rho a^{2} \Omega_{0}^{3}\right)$, we can see that our result is intermediate to that by L\&E and that by Burns \& Safronov (1973). Therefore, the simplifying assumption that the energy dissipation is a result of the bulge flexing made by Burns \& Safronov (1973) does not work well as stated by Efroimsky \& Lazarian (2000), but it is not so bad. Formula (39) in Purcell (1979) establishes $t=70\left(\mu Q / \rho a^{2} \Omega_{0}^{3}\right)$ when $\frac{c}{a}=0.5$, but it does not seem to be interesting to make any comparison because he derived that formula from an incorrect expression as stated above. Harris (1994), using data of Phobos, gave a value of 0.03 for the numerical factor in the Burns and Safronov formula, that is $t=33\left(\mu Q / \rho a^{2} \Omega_{0}^{3}\right)$, which is in excellent agreement with our results as Fig. 1 shows.

At this point, is necessary to say that the theory shown here like those developed by L\&E and Efroimsky \& Lazarian (2000) is, strictly speaking, applicable to oblate symmetric bodies only. In the general case of a triaxial body, the solution to the Euler equations are expressed in terms of elliptic functions instead of harmonic functions, the problem is more complicated, and even in the case of the prolate symmetric body the solution is not easy. The equations obtained for the oblate bodies are not applicable directly to prolate objects. This point is studied in detail in Efroimsky (2000) as well as in Efroimsky (2001). Then, one could think about the Comet Halley, which is clearly prolate in shape and has been chosen for application in this paper. As mentioned in the introduction, Comet Halley is a well-studied comet and it is in an excited rotational state, i.e. it is in a rotation with precession. This fortunate coincidence is a good reason why we will apply it to the expression of our study. Besides, for $\left\langle\cos ^{2} \theta>\right.$ not exceeding $\approx 1 / 7$, the oblatebody theory may be applied, as an estimation, to prolate bodies (see Sect. 7 in Efroimsky 2001). In the case of Comet P/Halley, $\theta$ is equal to $66^{\circ}$ (Belton et al. 1991) and therefore, the application of the theory can be considered as a fair approximation.

Comet Halley shows an irregular shape if it is considered in detail. However, if it is modelled as a simple geometric body then a symmetric ellipsoid is a good choice. As the largest dimension of Comet Halley is about $16 \mathrm{~km}$ and the transversal size is about $8 \mathrm{~km}$ (see for instance Sagdeev et al. 1986), we have chosen those dimensions as the values of the axis of our ellipsoid. Thus, and following with the notation used here, $a=b=4 \mathrm{~km}$ and $c=8 \mathrm{~km}$, and then the semi-axis ratio $\frac{c}{a}$ is 2 . Before continuing it is convenient to say that if the expressions of the previous sections are considered valid when the body is prolate $(c>a)$, we keep in mind that $h>1$, and then the sign of the right hand of the alignment rate equations have to be changed. While in the oblate case $\theta \rightarrow 0$ during the dissipative process, $\theta \rightarrow \frac{\pi}{2}$ when the body is prolate. In other words, when the minimum kinetic energy state has been reached, $\boldsymbol{\Omega}$ has the same direction as $\boldsymbol{L}$ and the $z$-axis (spin axis is parallel to the minimum principal axis of the body) if the body is oblate, and if the body is prolate $\boldsymbol{\Omega}$ has the same direction as $\boldsymbol{L}$ and the $x$-axis or $y$-axis (spin axis is also parallel to the minimum principal axis of the body) when such state is reached. Although at first glance this could seem a paradox, the relaxation time obtained from the shown expressions for $\frac{\mathrm{d} \theta}{\mathrm{d} t}$ is the same if, for example, $c=2 a$ or $a=2 c$, as can be proved. Then, the curves shown in Fig. 1 are the same curves changing the value of the $\frac{c}{a}$ ratio by their inverse values, i.e., the curve for $\frac{c}{a}=0.5$ is the same curve that we could obtain for $\frac{c}{a}=2$, and so on. Following our equations, the relaxation time for the Comet Halley goes from $33\left(\mu Q / \rho a^{2} \Omega_{0}^{3}\right)$ if the initial angle is $30^{\circ}$ to $39\left(\mu Q / \rho a^{2} \Omega_{0}^{3}\right)$ if the initial angle is near $90^{\circ}$. As stated before, Efroimsky \& Lazarian (2000) added a factor of 2 to their expression (39) in L\&E. They argued that the $W^{(\omega)}$ and $W^{(2 \omega)}$ should be averages but not amplitudes, but in fact they considered energies averaged in their preceding article, hence that factor of 2 should be not considered. So far we have included that factor in order to make more clear the comparison between the results of those authors and those derived from our expressions, however we think that our time relaxation results must be multiplied by 2 .

What about absolute numbers? There are two type of tasks if absolute numerical values are looked for. On the one hand, the knowledge of physical properties of the Comet Halley 
is needed. The parameters $\rho, \mu$ and $Q$ belong to this group. Probably the density is the least problematic quantity concerning its numerical value, (with regard to its spatial variability, if the Comet is homogeneous or it shows more or less porosity, the topic is more complicated) and it can be reasonably chosen as $10^{3} \mathrm{~kg} / \mathrm{m}^{3}$ for the purpose here looked for. However, the determination of the other two parameters is more difficult and, what is more, even speculative. Several estimations have been made from seismological data and, obviously neither pressure nor temperature conditions are appropriate for the case of this study. Estimations are $\mu=10^{10}-10^{11} \mathrm{~Pa}$ and $Q=100-1000$ (presently the values near 100 are believed to be more realistic) considering rocky materials such as silicates and carbonaceous forms. In fact, there are different factors that influence, particularly in $Q$ estimates such as chemical composition, graining, frequency, temperature, pressure, type of aggregation, humidity, porosity, structure of crack, surface state (see Efroimsky \& Lazarian 2000 and references therein).

In the case of Comet Halley we adopt the values suggested by Peale $\&$ Lissauer (1989), i.e. $\mu=10^{9} \mathrm{~Pa}$, and $Q<100$. Efroimsky (2001) suggested to take values appropriate not to solid ice but to dirty coarse-grained snow: $Q \sim 1$. At this point we can only invoke again the necessity to progress in laboratory work. The second type of task is related to the dynamical characteristics of the Comet Halley rotation. In the expression of the relaxation time there are two: $a$ and $\Omega_{0}$. In spite of the semi-axis $a$ appearing with exponent 2 , it would be absurd to have a more precise estimation than that here adopted of $4 \mathrm{~km}$, because of the errors that other parameters introduce in $t$ estimates. With regard to $\Omega_{0}$ (which appears in the relaxation time relation with exponent 3 ) the difficulty appears in the interpretation of the Comet Halley rotation. In fact, two periods (2.2 day and 7.4 day, see Belton 1991) were measured for the comet, and both periods must be considered, but the interpretation of those periods is not unique. Here we follow the model proposed by Belton et al. (1991) who established a $\theta$ angle of $66^{\circ}$ and a value of 3.7 day for the period of $\Omega_{0}$. With all the previous estimates, we determine values from $86\left(\theta_{0}=30^{\circ}\right)$ to $102\left(\theta_{0} \approx 90^{\circ}\right)$ million of years if $Q$ is equal to 1 , or $10^{4}$ million years if $Q$ is equal to 100 . Now, as the age of the Solar System is $4.6 \times 10^{9}$ years, we are not sure we can adopt the words written by Peale \& Lissauer (1989): "the decay time for a wooble of Halley's nucleus is long compared with the recorded history of the comet, but short compared with the age of the Solar System".

\section{Conclusions}

First, we establish here original expressions for the stress tensor components in the case of a free-torques symmetric ellipsoid body rotating in a complex state. Expressions for the components of the stress tensor were before proposed only for the case of a rectangular prism by $L \& E$. Our equations take into account the appropriate boundary conditions.

Following the approach of continuum mechanics, and introducing a physically reasonable approximation to that approach, we obtain values for the strain energy density and the alignment rate of a body rotating out of a principal inertia axis.
The importance of the double frequency mode, already noted by L\&E, has been indicated; even more, its relative importance with respect to the simple frequency mode is enhanced by a factor 2.5 in this work.

From that alignment rate we obtain the relaxation time values. Our values are higher that those obtained by $L \& E$ and are some lower than those shown by the equation given by Burn \& Safronov (1973). Application to the Comet P/Halley shows that an external cause (impact by other body or tidal actions) or effective outgassing processes have occurred after the Comet was incorporated to the Solar System.

Laboratory work on the physical parameter values are needed to progress in the tasks studied here. Particularly, the factor $Q$ shows an uncertainty of two orders of magnitude, which should be improved. This should be considered as a crucial point, since a relaxation time equal to the age of the Solar System would have very important implications.

In the course of our further research into the solar-system small-body population, we will get access to the physical parameters of comets and asteroids. This theory then should be applied to those objects, in order to better understand the history of their rotation states. On the other hand, the knowledge of rotation states may be the key to getting information about the bodies' physical parameters.

A typical relaxation time for a comet is an interval during which the spin state alters considerably (by dozens of angular degrees). Such an amount of relaxation may take millions of years. On the other hand, it has been shown in Efroimsky (2001) (and it also follows from Eq. (38) of our paper) that a small amount of relaxation (i.e., a small decrease in the nutation-cone half-angle) will take a much shorter time span. This makes the nutation-damping process potentially measurable by the presently available spacecraft-based equipment. This circumstance makes the nutation-damping theory applicable to planning future rendezvous missions and to interpreting their results.

Acknowledgements. We are very grateful to the reviewer, M. Efroimsky, who made a significant number of interesting suggestions, contributing to improve the manuscript. This work was supported by contracts PNE-001/2000-C-01 and AYA-2001-1177.

\section{Appendix}

Let $X, Y, Z$ be an inertial frame whose origin is the point $\mathrm{O}$ and let $x, y, z$ be another system which is rotating with angular velocity $\boldsymbol{\Omega}$. In principle, the point $\mathrm{O}$ is also assumed as the $x$, $y, z$ origin. From Classical Mechanics is known the relation

$\left(\frac{\mathrm{d} \boldsymbol{r}}{\mathrm{d} t}\right)_{\mathrm{i}}=\left(\frac{\mathrm{d} \boldsymbol{r}}{\mathrm{d} t}\right)_{\mathrm{b}}+\boldsymbol{\Omega} \times \boldsymbol{r}$

where the subscripts $i$ and $b$ indicates what the quantities are referred to in the inertial frame and the ligated body system (because a body rotating is assumed and this system is moving as the body does) and $\boldsymbol{r}$ can be any vector and, particularly the position vector (see, e.g. Marion 1970). If a second temporal derivation is made, the following expression is obtained:

$a_{\mathrm{i}}=\dot{\boldsymbol{\Omega}} \times \boldsymbol{r}_{\mathrm{b}}+2 \boldsymbol{\Omega} \times \boldsymbol{v}_{\mathrm{b}}+\boldsymbol{\Omega} \times\left(\boldsymbol{\Omega} \times \boldsymbol{r}_{\mathrm{b}}\right)$. 
Usually $\boldsymbol{\Omega}$ is assumed constant and then the first term of the right side of the above relation is missing. However, if $\dot{\boldsymbol{\Omega}}$ (time derivative of the angular velocity) is non zero this contribution has to be taken into account, which is seems was not considered by Purcell (1979). As the term $2 \boldsymbol{\Omega} \times \boldsymbol{v}_{\mathrm{b}}$ (Coriolis acceleration) is null, the relation is:

$$
\boldsymbol{a}_{\mathrm{i}}=\dot{\boldsymbol{\Omega}} \times \boldsymbol{r}_{\mathrm{b}}+\boldsymbol{\Omega} \times\left(\boldsymbol{\Omega} \times \boldsymbol{r}_{\mathrm{b}}\right) .
$$

Taking components in the first term of the vectorial equation, we have

$\dot{\boldsymbol{\Omega}} \times \boldsymbol{r}_{\mathrm{b}}=\left(\dot{\Omega}_{2} z-\dot{\Omega}_{3} y\right) \boldsymbol{i}+\left(\dot{\Omega}_{3} x-\dot{\Omega}_{1} z\right) \boldsymbol{j}+\left(\dot{\Omega} 1 y-\dot{\Omega}_{2} x\right) \boldsymbol{k}$,

$\boldsymbol{i}, \boldsymbol{j}$ and $\boldsymbol{k}$ being the unitary vectors in the body system.

From the equations of the symmetric top in free rotation, $\dot{\Omega}_{2}=\omega \Omega_{1}, \dot{\Omega}_{1}=-\omega \Omega_{2}, \dot{\Omega}_{3}=0,(\omega$ is angular velocity with which $\Omega$ is precessing around the $Z$-axis or around the $z$-axis), and the above equation can be written as

$$
\dot{\boldsymbol{\Omega}} \times \boldsymbol{r}_{\mathrm{b}}=\omega z \Omega_{1} \boldsymbol{i}+\omega z \Omega_{2} \boldsymbol{j}+\left(-\omega y \Omega_{2}-\omega x \Omega_{1}\right) \boldsymbol{k} .
$$

Since $\boldsymbol{\Omega} \times\left(\boldsymbol{\Omega} \times \boldsymbol{r}_{\mathrm{b}}\right)=\boldsymbol{\Omega}\left(\boldsymbol{\Omega} . \boldsymbol{r}_{\mathrm{b}}\right)-\boldsymbol{r}_{\mathrm{b}} \Omega^{2}$,

$$
\begin{aligned}
\boldsymbol{\Omega} \times\left(\boldsymbol{\Omega} \times \boldsymbol{r}_{\mathrm{b}}\right)= & {\left[\Omega_{1}\left(\Omega_{1} x+\Omega_{2} y+\Omega_{3} z\right)-x \Omega^{2}\right] \boldsymbol{i} } \\
& +\left[\Omega_{2}\left(\Omega_{1} x+\Omega_{2} y+\Omega_{3} z\right)-y \Omega^{2}\right] \boldsymbol{j} \\
& +\left[\Omega_{3}\left(\Omega_{1} x+\Omega_{2} y+\Omega_{3} z\right)-z \Omega^{2}\right] \boldsymbol{k} .
\end{aligned}
$$

Now, and after writing the components of the angular velocity as a function of the $\Omega_{\perp}$ by means of the relations $\Omega_{1}=$ $\Omega_{\perp} \cos \omega t, \Omega_{2}=\Omega_{\perp} \sin \omega t, \Omega_{3}=$ constant, we write the following expression for the acceleration:

$$
\begin{aligned}
\boldsymbol{a}_{\mathrm{i}}= & \left(-x \Omega_{\perp}^{2} \sin ^{2} \omega t-x \Omega_{3}^{2}+y \Omega_{\perp}^{2} \sin \omega t \cos \omega t\right. \\
& \left.+z \Omega_{3} \Omega_{\perp} \cos \omega t+z \omega \Omega_{\perp} \cos \omega t\right) \boldsymbol{i} \\
& +\left(x \Omega_{\perp}^{2} \sin \omega t \cos \omega t-y \Omega_{\perp}^{2} \cos ^{2} \omega t-y \Omega_{3}^{2}\right. \\
& \left.+z \Omega_{\perp}^{2} \sin \omega t+z \omega \Omega_{\perp} \sin \omega t\right) \boldsymbol{j} \\
& +\left(x \Omega_{\perp} \Omega_{3} \cos \omega t+y \Omega_{\perp} \Omega_{3} \sin \omega t\right. \\
& \left.-z \Omega_{\perp}^{2}-\omega \Omega_{\perp} x \cos \omega t-\omega \Omega_{\perp} y \sin \omega t\right) \boldsymbol{k} .
\end{aligned}
$$

Now we look only at the first component. The first term can be written as $-\frac{\Omega_{\perp}^{2} x}{2}+\frac{\Omega_{\perp}^{2} x}{2}\left(\frac{1-2 \sin ^{2} \omega t}{2}\right)$, and since $\omega=(h-1) \Omega_{3}$ the last term of the first component is $z \Omega_{3} \Omega_{\perp}(h-1) \cos \omega t$. Thus,

$$
\begin{aligned}
a_{i 1}= & \left(-\frac{\Omega_{\perp}^{2}}{2}+\Omega_{3}^{2}\right) x+\frac{\Omega_{\perp}^{2} x}{2}\left(\frac{1-2 \sin ^{2} \omega t}{2}\right) \\
& +y \Omega_{\perp}^{2} \sin \omega t \cos \omega t \\
& +z \Omega_{3} \Omega_{\perp} \cos \omega t+z \Omega_{3} \Omega_{\perp}(h-1) \cos \omega t .
\end{aligned}
$$

The first term is independent of time and we are only interested in the terms dependent on time, which are written as

$$
\begin{aligned}
a_{i 1_{t}}= & \frac{\Omega_{\perp}^{2} x}{2}\left(\frac{1-2 \sin ^{2} \omega t}{2}\right)+y \Omega_{\perp}^{2} \sin \omega t \cos \omega t \\
& +z \Omega_{3} \Omega_{\perp} \cos \omega t \\
& +z \Omega_{3} \Omega_{\perp}(h-1) \cos \omega t .
\end{aligned}
$$

As the before last term is canceled with part of the last term and, as $\cos 2 \omega t=\frac{1-2 \sin ^{2} \omega t}{2}$ and $\sin 2 \omega t=2 \sin \omega t \cos \omega t$, we can write

$a_{i 1_{t}}=\frac{1}{2} \Omega_{\perp}^{2} x \cos 2 \omega t+\frac{1}{2} \Omega_{\perp}^{2} y \sin 2 \omega t+z \Omega_{3} \Omega_{\perp} h \cos \omega t$.

In a similar way, the other two components can be obtained as:

$a_{i 2_{t}}=\frac{1}{2} \Omega_{\perp}^{2} x \sin 2 \omega t-\frac{1}{2} \Omega_{\perp}^{2} y \cos 2 \omega t+z \Omega_{3} \Omega_{\perp} h \sin \omega t$

$a_{i 3_{t}}=\Omega_{\perp} \Omega_{3}(2-h)(x \cos \omega t+y \sin \omega t)$,

which are written in the same form as equations already found by L\&E (Eq. (19)).

Now it is easy to show that the components of the acceleration are obtained by the derivatives of the stress tensor given in the text. In fact, the equilibrium equation (from the continuum equation of motion) is $\rho F_{i}=-\frac{\partial \tau_{i j}}{\partial x_{j}}$. Therefore, as

$$
\begin{aligned}
\tau_{x x}= & \frac{\rho}{4} \Omega_{\perp}^{2}\left[x^{2}-\left(a^{2}-\frac{a^{2}}{c^{2}} z^{2}-y^{2}\right)\right] \cos 2 \omega t \\
\tau_{x y}= & \frac{\rho}{4} \Omega_{\perp}^{2}\left[y^{2}-\left(a^{2}-\frac{a^{2}}{c^{2}} z^{2}-x^{2}\right)\right] \sin 2 \omega t \\
\tau_{x z}= & \frac{\rho}{2} \Omega_{\perp} \Omega_{3} h\left[z^{2}-\left(a^{2}-x^{2}-y^{2}\right) \frac{c^{2}}{a^{2}}\right] \cos \omega t \\
\tau_{y y}= & -\frac{\rho}{4} \Omega_{\perp}^{2}\left[y^{2}-\left(a^{2}-\frac{a^{2}}{c^{2}} z^{2}-x^{2}\right)\right] \cos 2 \omega t \\
\tau_{y z}= & \frac{\rho}{2} \Omega_{\perp} \Omega_{3} h\left[z^{2}-\left(a^{2}-x^{2}-y^{2}\right) \frac{c^{2}}{a^{2}}\right] \sin \omega t \\
\tau_{z z}= & \rho \Omega_{\perp} \Omega_{3}\left(2-h \frac{c^{2}}{a^{2}}-h\right)(x \cos \omega t+y \sin \omega t) \\
& \times\left\{z-\left[\left(a^{2}-x^{2}-y^{2}\right) \frac{c^{2}}{a^{2}}\right]^{\frac{1}{2}}\right\}
\end{aligned}
$$

the components of the accelerations will be:

$$
\begin{aligned}
a_{i 1_{t}}= & \frac{1}{\rho}\left(\frac{\partial \tau_{x x}}{\partial x}+\frac{\partial \tau_{x y}}{\partial y}+\frac{\partial \tau_{x z}}{\partial z}\right)=\frac{1}{2} \Omega_{\perp}^{2} x \cos 2 \omega t \\
& +\frac{1}{2} \Omega_{\perp}^{2} y \sin 2 \omega t+z \Omega_{3} \Omega_{\perp} h \cos \omega t \\
a_{i 2_{t}}= & \frac{1}{\rho}\left(\frac{\partial \tau_{y x}}{\partial x}+\frac{\partial \tau_{y y}}{\partial y}+\frac{\partial \tau_{y z}}{\partial z}\right)=\frac{1}{2} \Omega_{\perp}^{2} x \sin 2 \omega t \\
& -\frac{1}{2} \Omega_{\perp}^{2} y \cos 2 \omega t+z \Omega_{3} \Omega_{\perp} h \sin \omega t
\end{aligned}
$$

$a_{i 3_{t}}=\frac{1}{\rho}\left(\frac{\partial \tau_{z x}}{\partial x}+\frac{\partial \tau_{z y}}{\partial y}+\frac{\partial \tau_{z z}}{\partial z}\right)=\Omega_{\perp} \Omega_{3}(2-h)$

$$
\times(x \cos \omega t+y \sin \omega t),
$$

as it is easy to prove. 


\section{References}

Belton, M. J. S., Julian, W. H., Anderson, A. J., \& Mueller, B. E. A. 1991, Icarus, 93, 183

Burn, J. A., \& Safronov, V. S. 1973, MNRAS, 165, 403

Durelli, A. J., Phillips, E. A., \& Tsao, C. H. 1958, Introduction to the Theoretical and Experimental Analysis of Stress and Strain (McGraw Hill Book Co.)

Efroimsky, M. 2000, J. Math. Phys., 41, 1854

Efroimsky, M. 2001, P\&SS, 49, 937

Efroimsky, M. 2002, Adv. Space Res., 29, 725

Efroimsky, M., \& Lazarian, A. 2000, MNRAS, 311, 269
Harris, A. W. 1994, Icarus, 107, 209

Lazarian, A., \& Efroimsky, M. 1999, MNRAS, 303, 673

Marion, J. B. 1970, Classical Dynamics of Particles and Systems (Academis Press, New York)

Peale, S. J., \& Lissaueer, J. J. 1989, Icarus, 79, 396

Prendergast, K. H. 1958, AJ, 63, 412

Purcell, E. M. 1979, AJ, 231, 404

Sedov, L. I. 1971, A course in continuum mechanics (Wolters-Noordhoff Publishing, Groningen)

Sokolnikoff, S. I. 1956, Mathematical Theory of Elasticity, second edition (McGraw-Hill, New York) 